Research Article

Open Access

\title{
Health Benefits of Dietary Tree Peony Seed Oil in a High Fat Diet
}

\section{Hamster Model}

Zhiqiang Zheng ${ }^{2}$, Jigang Han ${ }^{1,3}$, Yingyi Mao ${ }^{2}$, Xue Tang ${ }^{1,3}$, Yan Guan ${ }^{2, \#, ~ Y o n g h o n g ~ H u ~}{ }^{1,3,{ }^{*}}$

${ }^{1}$ Shanghai Key Laboratory of Plant Functional Genomics and Resources, Shanghai Chenshan Plant Science Research Center (CAS), Chenshan Botanical Garden, Shanghai, 201602, China;

${ }^{2}$ Abbott Nutrition Research \& Development Center, Shanghai, 201203, China; ${ }^{3}$ Shanghai Institute of Jiangnan Tree Peony, Shanghai, 201602, China

\#Co-corresponding author: Yan Guan, MD, 3F, No. 2 Building, 476 Li Bing Road, Zhangjiang High-Tech Park, Shanghai, 201203, China

*Co-corresponding author: Yonghong Hu, Ph.D., Professor, Shanghai Chenshan Botanical Garden, 3888 Chenhua Road, Shanghai, 201602, China

Submission Date: October 14, 2016, Acceptance date: February 24, 2017: Publication date: February 28, 2017

Citation: Zheng Z, Han J, Mao Y, Tang X, Guan Y, Hu Y., Health Benefits of Dietary Tree Peony Seed Oil in a High Fat Diet Hamster Model. Functional Foods in Health and Disease 2017; 7(2): 135-148

\begin{abstract}
Background: Tree peony (Paeonia ostii) seed oil is rich in different unsaturated fatty acids, including monounsaturated fatty acids (MUFA), n-3, and n-6 polyunsaturated fatty acids (PUFA). Overall, health benefits of this edible plant oil have not been widely explored. In this study, we experimentally investigated benefits of dietary tree peony seed oil (PSO) in dyslipidemia-associated metabolic diseases using a high fat diet hamster model.
\end{abstract}

Methods: High fat diets (HFD) containing $15 \%$ coconut oil (CO) or PSO were initially developed based on the rodent chow diet. Fatty acid profiles of diets and red blood cells (RBC) from animals fed these diets for 8 weeks were analyzed and compared. Effects of these oil supplements on triglycerides and cholesterol levels were characterized. Benefits on fatty liver progress were also investigated in this animal model.

Results: HFD fortified with $15 \%$ PSO was abundant in different unsaturated fatty acids, containing $40 \% \alpha$-linolenic acid, 27\% linoleic acid, and $23 \%$ oleic acid respectively. Compared 
to the control group with $15 \% \mathrm{CO}$, animals with $15 \%$ PSO displayed dramatic alteration of in vivo fatty acid profile in RBC, featuring a significant increase in $n-3$ but no change in $n-6$ PUFA, thereby resulting in a decreased ratio of n-6 to n-3 PUFA. PSO intervention also remarkably reduced triglyceride levels in both blood and adipose tissues, but did not affect circulating cholesterol. Moreover, benefits on liver health were observed in the PSO group, evidenced with reduced hepatic steatosis and improved hepatic histology.

Conclusion: Altogether, the data demonstrated multifaceted benefits of dietary PSO in reducing important risk factors of dyslipidemia-associated cardiovascular and liver diseases.

Keywords: Peony seed oil, High fat diet, Triglyceride, Cholesterol, Hepatic steatosis

\section{BACKGROUND:}

With a cultivation history over 2000 years in many regions, the Chinese tree peony belongs to the genus Paeonia. Currently, there are over 1000 tree peony cultivars in China alone [1]. Besides its outstanding ornamental value, peony has also been long used in Chinese medicine. Significantly, in recent years there has been an increasing interest in peony seeds, which is estimated to have an annual yield of 57,855 tons [2]. To date, many kinds of bioactive ingredients, including unsaturated fatty acids, tocopherols, and phenolic and monoterpene glycosides have been found to be rich in the dark oval seeds of peony [3]. Especially prominent is the uncommonly high level of unsaturated fatty acids (> 90\%) in the seed [2, 4]. Usually containing about 25\% monounsaturated fatty acids (MUFA), the seed has nearly 65\% essential polyunsaturated fatty acids (PUFA), comprising approximately $40 \% \alpha$-linolenic acid and 25\% and linoleic acid respectively $[2,4,5]$. The level of $\alpha$-linolenic acid in most edible oils is less than 3\% [6]. Such high levels of n-3 PUFA in peony seed oil (PSO) are generally uncommon. As an essential fatty acid, $\alpha$-linolenic acid cannot be synthesized by the human body and has to be obtained from diets. Ascribed to the property of unusually high levels of $\alpha$-linolenic acid, in recent years PSO has attracted extensive research interest in an effort to develop a new source of $\alpha$-linolenic acid-rich edible oil and to meet the ever increasing demand in the food industry, especially in China.

Nowadays, the increasing prevalence of obesity-associated metabolic diseases is one of the global major health threats. As a classical feature, dyslipidemia is closely linked to increased risks of non-alcoholic liver disease (NAFLD), cardiovascular diseases (CVD), type 2 diabetes, and more. Some dietary factors, including the excess intake of saturated fats, play adverse roles in the occurrence of dyslipidemia. Abundant evidence has suggested a strong correlation between the overconsumption of saturated fats and dyslipidemia, manifested with increased blood low-density lipoprotein (LDL), triglyceride and free fatty acids, decreased high-density lipoprotein (HDL), and more [7]. Similarly, the excess intake of saturated fats can lead to fat 
accumulation within liver and exacerbates progression of NAFLD [8, 9]. Although the pathogenic mechanisms involved in hepatic lipid accumulation are not fully understood, liver steatosis may result from impaired balance between lipid availability and clearance in the liver. Classical western diets are characterized with high ratio of n-6 to n-3 PUFA, ranging from 15:1 to 20:1, which is suggested to involve in pathogenesis of some metabolic diseases, cancer, and inflammatory diseases [10-12]. According to the United Nations Food and Agriculture Organization and the World Health Organization, the recommended ratio of n-6 to n-3 PUFA is less than 5 [13]. Previous evidence indicated that unsaturated fats, especially n-3 polyunsaturated fats, may help ameliorate dyslipidemia and could have protective effects against medical conditions like NAFLD and CVD [14, 15]. Replacement of saturated fat with polyunsaturated fat has been shown to decrease total cholesterol and LDL-cholesterol by lowering production rates and increasing clearance rates of LDL-cholesterol. In patients with NAFLD, PUFA levels are significantly lower while also having a less favorable higher ratio of n-6 to n-3 PUFA [16]. Therefore, a lower ratio of n-6 to n-3 PUFA is more desirable in reducing risks of these diseases [16-18]. The ratio of n-6 to n-3 PUFA in PSO can be as low as around 0.6, which is much lower than the values of other important edible oils [2].

Although rich in different unsaturated fatty acids, PSO as a whole has not been evaluated in terms of reducing risk factors of different metabolic diseases. Focusing on dyslipidemia-associated CVD and NAFLD, in this study we experimentally investigated health benefits of PSO supplement in an adult hamster model, which was fed high fat diets (HFD) comprising $15 \%$ fat and $0.1 \%$ cholesterol for 8 weeks. Hamsters have long been used as an animal model to assess HFD-induced cardiovascular diseases [19]. Compared to other rodents, this animal species has a low rate of endogenous cholesterol synthesis, cholesteryl ester transfer protein activity and apo B protein secretion from liver and intestine [20, 21]. Furthermore, like humans, hamsters take up about $80 \%$ of LDL via LDL receptor pathway [22]. Therefore, hamsters fed high cholesterol and saturated fat diets respond more similarly to humans in lipoprotein metabolism than other rodents.

\section{METHODS:}

PSO preparation. Tree peony (Paeonia ostii) seeds were collected from Bozhou, Anhui Province, China. Briefly, the raw oil was first extracted by mechanic pressing (High pressure oil pressing machine Type W, KEST-ENG Co., Ltd.) under conditions of 60 Mpa for 30 minutes. Then, the raw oil was subjected to standard refining processes, including degumming $(0.3 \%$ phosphoric acid, food grade), deacidification $\left(85^{\circ} \mathrm{C}\right.$ for 15 minutes), bleaching $\left(105{ }^{\circ} \mathrm{C}\right.$ for 25 minutes), and deodorization $\left(240{ }^{\circ} \mathrm{C}\right.$ for 90 minutes). No antioxidants were applied through the whole process of oil preparation.

Animals and diet information. All experimental protocols were approved by Abbott Animal 
Welfare Committee and HD Biosciences Institutional Animal Care and Use Committee. 8 weeks old male Golden-Syrian hamsters (Beijing Vital River Laboratories Co., Ltd.) were group housed, fed respective diets, and provided with water ad libitum. Animals were maintained on a $12 \mathrm{~h} / 12 \mathrm{~h}$ light/dark cycle in an AAALAC accredited animal facility with controlled temperature $\left(22 \pm 3{ }^{\circ} \mathrm{C}\right)$ and humidity $(55 \pm 15 \%)$.

Three groups of animals ( $n=10$ each group) were included in this study and subjected to three different dietary interventions for 8 weeks. These diets were named as CHOW, HFD-CO, and HFD-PSO, containing 5\% soybean oil, 15\% coconut oil (CO) and 15\% PSO respectively. Detailed compositions of these diets were listed in Table 1.

Fatty acid profile analysis. Analysis of fatty acid profiles of diets and red blood cells (RBC) were described previously [23]. Briefly, lipids of RBC were extracted using isopropanol and hexane. Then, a portion of the extract was subjected to base-catalyzed transesterification, which converted the phospholipid acyl chains to fatty acid methyl esters. Individual fatty acids were separated by gas-liquid chromatography and then quantified using a standard curve from commercially purchased standards (GLC746, Nu-Chek Prep., Inc.).

Table 1. Diet Compositions

\begin{tabular}{llll}
\hline Ingredients & $\begin{array}{l}\text { CHOW (5\% } \\
\text { soybean oil) }(\mathbf{g})\end{array}$ & $\begin{array}{l}\text { HFD-CO (15\% } \\
\text { coconut oil) })\end{array}$ & $\begin{array}{l}\text { HFD-PSO (15\% } \\
\text { peony seed oil) (g) }\end{array}$ \\
\hline Casein & 200 & 200 & 200 \\
L-cystein & 3 & 3 & 3 \\
Corn starch & 442.49 & 249 & 249 \\
Maltodextrin 10 & 132 & 132 & 132 \\
Sucrose & 100 & 100 & 100 \\
Cellulose BW200 & 50 & 50 & 50 \\
Oils & 50 & 136 & 136 \\
tert-butylhydroquinone & 0.014 & 0.04 & 0.04 \\
Mineral mix S10022G & 35 & 35 & 35 \\
Vitamin mix V10037 & 10 & 10 & 10 \\
Choline Bitartrate & 2.5 & 2.5 & 2.5 \\
Cholesterol & 0 & 0.92 & 0.92 \\
Total & 1025 & 918.46 & 918.46 \\
\hline
\end{tabular}

Analysis of triglyceride and cholesterol. Hamsters were fasted overnight. Blood was withdrawn from hamsters after 8 weeks of dietary treatments and serum was prepared accordingly. Lipid extract were prepared using epididymal adipose sample as well. Lipid panels (total triglyceride, total cholesterol, HDL-Cholesterol, LDL-Cholesterol) were then analyzed using the Hitachi 7080 automatic biochemical analyzer (Japan Hitachi Trade Co., Ltd.). 
Liver histological analysis. All animals were sacrificed by $\mathrm{CO}_{2}$ inhalation and liver tissues were collected at the end of experiment. Liver tissue from the left lobe was removed and divided into 2 parts. Each part was used for hematoxylin and eosin (H\&E) analysis and oil red staining respectively. Liver tissues were evaluated and scored for hepatic steatosis according to the following criteria: grade 0 , no fat; grade 1, steatosis occupying less than $33 \%$ of the hepatic parenchyma; grade 2,34-66\% of the hepatic parenchyma; grade 3, more than $66 \%$ of the hepatic parenchyma [24].

Statistical analysis. Results were expressed as mean \pm standard error of the mean (SEM). Statistical analysis was performed using GraphPad Prism 6 with one way analysis of variance (ANOVA) followed by Turkey multiple comparison test. The difference was considered statistically significant when $\mathrm{p}$ value is less than 0.05 .

\section{RESULTS:}

Fatty acid profiles. In this study, two diets named HFD-CO and HFD-PSO, were initially developed by fortifying the chow with $15 \% \mathrm{CO}$ and PSO respectively. Additionally, these HFD were also fortified with $0.1 \%$ cholesterol. Detailed diet compositions were listed in Table 1. All diets were then subjected to fatty acid profile analysis and results were showed in Table 2. As expected, HFD-CO showed a classical lipid profile rich in saturated fatty acids, exceeding more than $90 \%$ of total fats. In contrast, both CHOW and HFD-PSO had much lower levels of saturated fatty acids, accounting for $17 \%$ and $9 \%$ respectively. Replacing CO with PSO in HFD resulted in a fatty acid profile similar to the raw PSO, containing $23 \%$ oleic acid (C18:1n-9), 27\% linoleic acid (C18:2n-6), and 40\% $\alpha$-linolenic acid (C18:3n-3) respectively (Table 2).

After 8 weeks of dietary intervention, the in vivo fatty acid profiles of RBC were analyzed and the result was summarized in Table 3. RBC from the HFD-PSO group showed the highest level of n-3 PUFA, containing $2.21 \%$ of $\alpha$-linolenic acid, which were 44 folds of that from HFD-CO. However, in contrast to the evident increase in n-3 PUFA, the n-6 PUFA level in the HFD-PSO group was similar to that of HFD-CO, even though its linoleic acid level increased by 40\%. Moreover, the MUFA level in RBC of HFD-PSO was slightly lower than HFD-CO, although the diet contained much higher levels of oleic acid. These data suggested that even though the diets were rich in different classes of unsaturated fatty acids, PSO was most effective in enriching n-3 PUFA in vivo, particularly $\alpha$-linolenic acid, which had little to no effect on changing total n-6 PUFA level and MUFA. Accordingly, the group of HFD-PSO showed the lowest ratio of n-6 to n-3 PUFA in vivo, only accounting for about 44\% of HFD-CO and CHOW. 


\begin{tabular}{llll}
\hline \multicolumn{2}{l}{ Table 2. Fatty Acid Profiles of Diets } & & \\
\hline Fatty acids & CHOW $(\%)$ & HFD-CO $(\%)$ & HFD-PSO $(\%)$ \\
\hline C8:0 & 0.07 & 7.91 & 0.02 \\
C10:0 & 0.12 & 5.92 & 0.05 \\
C12:0 & 0.28 & 44.46 & 0.07 \\
C14:0 & 0.52 & 16.34 & 0.20 \\
C15:0 & 0.07 & 0.04 & 0.05 \\
C16:0 & 11.73 & 8.72 & 6.53 \\
C16:1n-7 & 0.12 & 0.02 & 0.11 \\
C17:0 & 0.12 & 0.02 & 0.10 \\
C18:0 & 4.24 & 9.01 & 2.10 \\
C18:1n-9 & 23.23 & 2.46 & 23.15 \\
C18:2n-6 & 51.84 & 3.73 & 26.79 \\
C18:3n-3 & 6.68 & 0.47 & 40.31 \\
C20:0 & 0.33 & 0.13 & 0.17 \\
C20:1 n-9 & 0.17 & UD & 0.29 \\
C22:0 & 0.33 & 0.04 & 0.05 \\
C22:5 n-3 & UD & UD & UD \\
C24:0 & UD & UD & UD \\
C24:1 & 0.02 & UD & UD \\
\hline C8:0: & & &
\end{tabular}

C8:0: aprylic acid; C10:0: capric acid; C12:0: lauric acid; C14:0: myristic acid; C15:0: pentadecylic acid; C16:0: palmitic acid; C16:1n-7: palmitoleic acid; C17:0: margaric acid; C18:0: stearic acid; C18:1n-9: oleic acid; C18:2n-6: linoleic acid; C18:3n-3: $\alpha$-linolenic acid; C20:0: arachidic acid; C20:1n-9: gondoic acid; C22:0: behenic acid; C22:5n-3: docosapentaenoic acid; C24:0: lignoceric acid; C24:1: nervonic acid; UD: undetectable.

Table 3. Summary of Fatty Acid Profiles of Red Blood Cells

\begin{tabular}{lccc}
\hline Fatty acids & CHOW $(\boldsymbol{\%})$ & HFD-CO $(\boldsymbol{\%})$ & HFD-PSO $(\boldsymbol{\%})$ \\
\hline saturated & 46.68 & 46.17 & 45.88 \\
unsaturated & 53.29 & 53.86 & 54.13 \\
monounsaturated & 15.81 & 18.09 & 14.89 \\
polyunsaturated & 37.48 & 35.78 & 39.25 \\
n-3 PUFA & 4.47 & 4.22 & 9.18 \\
C18:3n-3 & UD & 0.05 & 2.21 \\
C20:5n-3 & UD & UD & UD \\
C22:5n-3 & 0.99 & 0.71 & 3.43 \\
C22:6n-3 & 3.48 & 3.46 & 3.54 \\
n-6 PUFA & 33.01 & 31.56 & 30.07 \\
C18:2n-6 & 13.31 & 11.86 & 16.69 \\
C20:4n-6 & 14.81 & 14.61 & 10.97 \\
C22:4n-6 & 2.97 & 2.55 & 1.22 \\
Ratio of n-6 to n-3 & 7.38 & 7.48 & 3.28 \\
\hline
\end{tabular}

C18:3n-3: $\alpha$-linolenic acid; C20:5n-3: eicosapentaenoic acid; C22:5n-3: docosapentaenoic acid; C22:6n-3: docosahexaenoic acid; C18:2n-6: linoleic acid; C20:4n-6: arachidonic acid; C22:4n-6: adrenic acid; UD: undetectable. 
Body weight gain. Relative to $5 \%$ fat in $\mathrm{CHOW}$ diet, both HFD-CO and HFD-PSO contained much higher levels of fats $(15 \%)$, resulting in higher energy density in high fat diets $(4.4 \mathrm{kcal} / \mathrm{g})$ than the CHOW diet $(3.9 \mathrm{kcal} / \mathrm{g})$. Through the whole intervention process, food intake in the three groups of animals was not different compared to each other (data not shown). As demonstrated in Figure 1, HFD-CO and HFD-PSO had a larger body weight gain than CHOW, due to relative higher energy intake from HFD. However, during the 8 weeks dietary intervention, no difference in body weight gain was observed between HFD-CO and HFD-PSO (Figure 1).

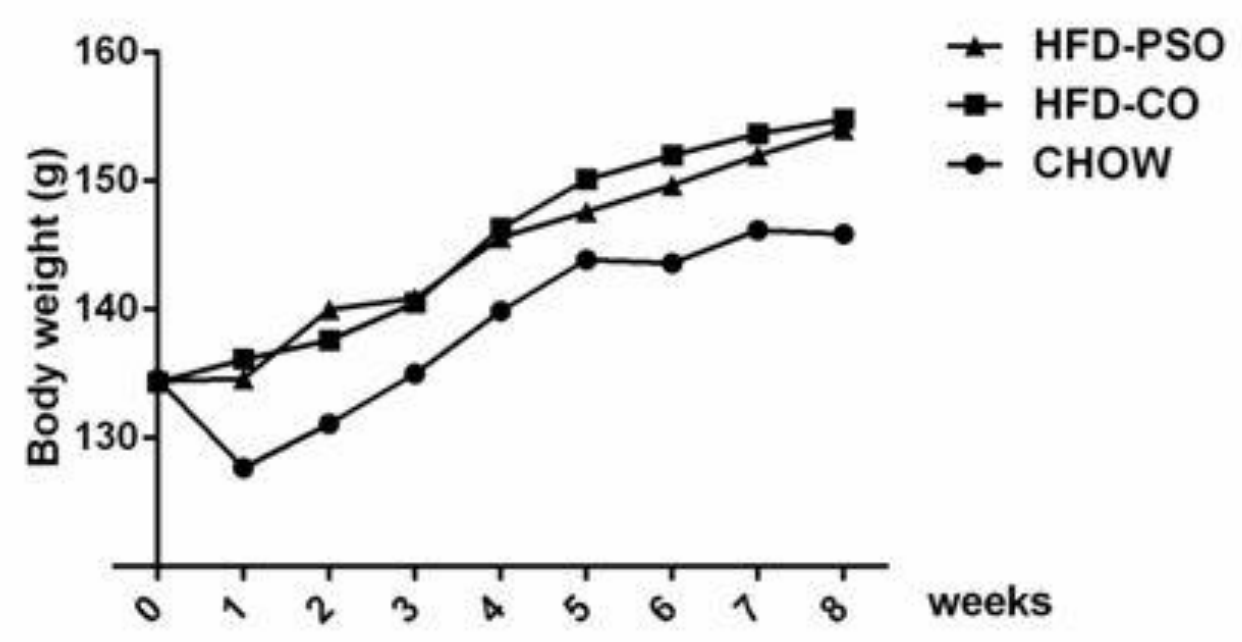

Figure 1. Animal Body Weight Gain. Three groups of adult hamsters ( $n=10$ for each group) were fed with diets of CHOW, HFD-CO and HFD-PSO for 8 weeks, respectively. CHOW: chow diet; HFD-CO: chow diet fortified with $15 \%$ coconut oil and $0.1 \%$ cholesterol; HFD-PSO: chow diet fortified with $15 \%$ peony seed oil and $0.1 \%$ cholesterol. Animal body weights were recorded weekly and compared to each other.

Triglyceride and cholesterol levels. Triglyceride levels in both blood and adipose tissues were then analyzed. Compared to $\mathrm{CHOW}$, plasma, and epididymal adipose triglycerides in the HFD-CO group were nearly doubled. When compared to HFD-CO, there was a remarkable reduction in both plasma and epididymal adipose triglycerides observed in HFD-PSO (Figure 2 $\mathrm{A}$ and B), accounting for $60 \%$ and $35 \%$ respectively. In terms of circulating cholesterol, incorporation of $0.1 \%$ cholesterol into HFD-CO and HFD-PSO diets strongly raised both LDL-cholesterol and HDL-cholesterol in blood (Figure $2 \mathrm{C}$ and D). However, no difference in circulating LDL-cholesterol and HDL-cholesterol was observed between groups of HFD-CO and HFD-PSO, thereby suggesting PSO supplement had no effect on circulating cholesterol levels.

Hepatic steatosis. HFD is one of the most common ways to induce NAFLD in animal models. In this study, the liver tissue in the CHOW group demonstrated no signs of lipid accumulation 
with a normal structure of hepatic lobules (Figure 3 A). However, HFD-CO treatment resulted in accumulation of abundant lipid droplets within the liver tissue. Moreover, it also caused significant vacuolar degeneration of hepatocytes, disruption of normal hepatic lobules and infiltration of inflammatory cells (Figure $3 \mathrm{~A}$ ), and massive hepatic steatosis, which was quantified in Figure 3 B. Compared to HFD-CO, HFD-PSO treatment significantly improved liver histology, characterized with less lipid droplet accumulation, fewer destroyed structures of liver lobule, and less hepatic steatosis (Figure $3 \mathrm{~A}$ and B).

A

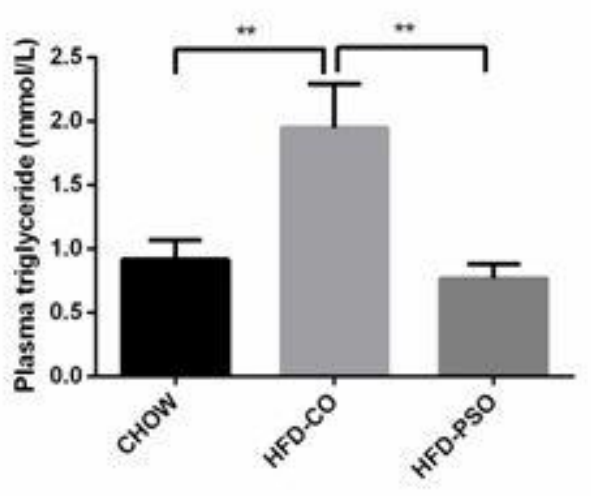

C

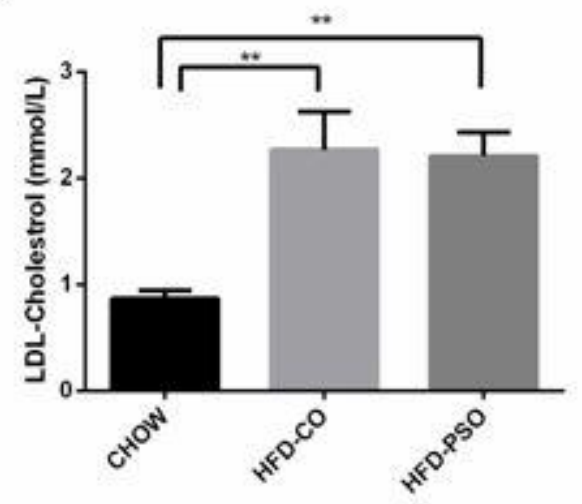

B

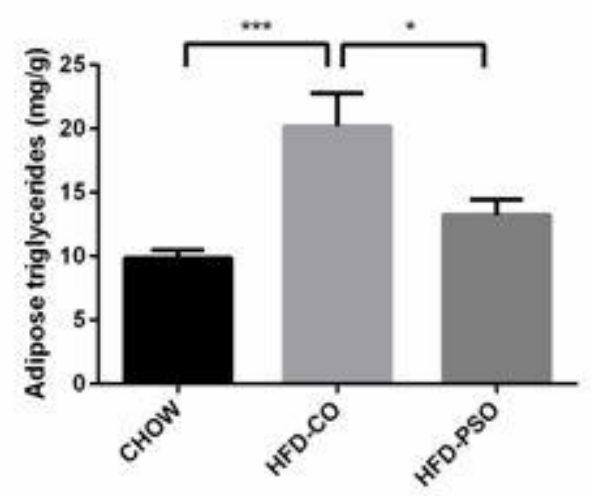

D

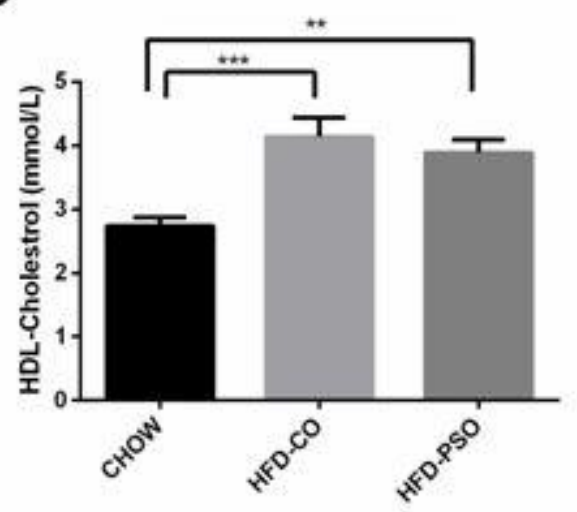

Figure 2. Effects of PSO Supplement on Triglycerides and Cholesterol Levels. Three groups of adult hamsters ( $\mathrm{n}=10$ for each group) were fed with diets of CHOW, HFD-CO and HFD-PSO for 8 weeks, respectively. CHOW: chow diet; HFD-CO: chow diet fortified with $15 \%$ coconut oil and $0.1 \%$ cholesterol; HFD-PSO: chow diet fortified with $15 \%$ peony seed oil and $0.1 \%$ cholesterol. Plasma and epididymal adipose triglycerides levels were analyzed and showed in $\mathbf{A}$ and $\mathbf{B}$ respectively. Plasma LDL-cholesterol and HDL-cholesterol were analyzed and showed in $\mathbf{C}$ and $\mathbf{D}$ respectively. Statistical analysis was performed using one-way ANONA analysis followed by Turkey multiple comparison test. *: $\mathrm{p}<0.05, * *$ : $\mathrm{p}<0.01, * * *$ : $<<0.001$. 

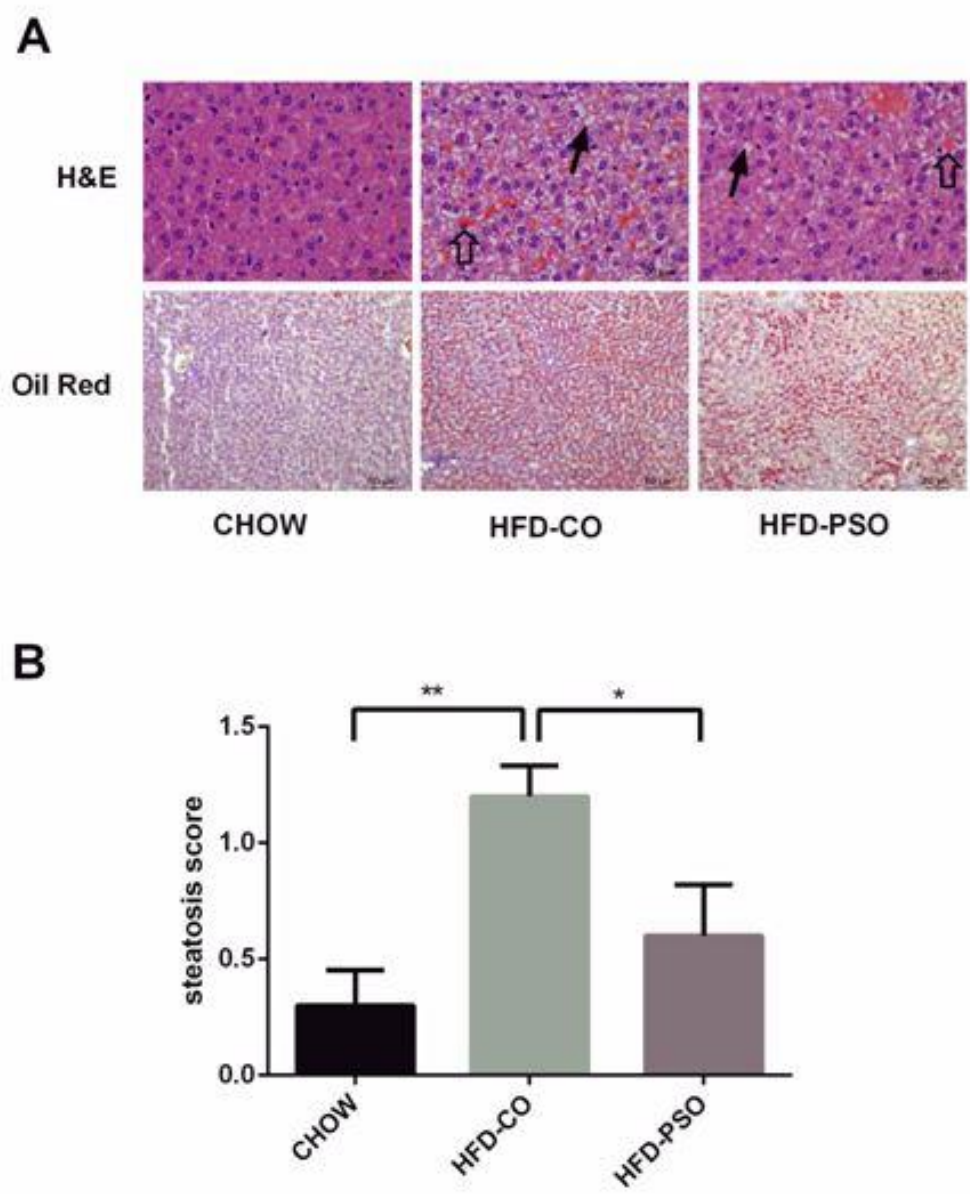

Figure 3. Effects of PSO Supplement on None-alcoholic Fatty Liver Disease. Three groups of adult hamsters ( $\mathrm{n}=10$ for each group) were fed with diets of CHOW, HFD-CO and HFD-PSO for 8 weeks, respectively. CHOW: chow diet; HFD-CO: chow diet fortified with $15 \%$ coconut oil and $0.1 \%$ cholesterol; HFD-PSO: chow diet fortified with $15 \%$ peony seed oil and $0.1 \%$ cholesterol. A. Histological images of liver tissue sections subjected to H\&E and oil red staining respectively. Representative lipid droplets and infiltrated inflammatory cells were marked with $\uparrow$ and $\uparrow$ respectively. B. Hepatic steatosis score for each group was quantified according to following criteria: grade 0, no fat; grade 1, steatosis occupying less than $33 \%$ of the hepatic parenchyma; grade 2, 34-66\% of the hepatic parenchyma; grade 3, more than $66 \%$ of the hepatic parenchyma. Statistical analysis was performed using one-way ANOVA analysis followed by Turkey multiple comparison test. *: $\mathrm{p}<0.05, * *$ : $<0.01$.

\section{DISCUSSION:}

In recent years, PSO has gained increasing popularity as a good resource in food and nutraceutical industries. Compared to other common plant oils, including soybean, peanut, corn, and sunflower, PSO contains very high levels of different unsaturated fatty acids, including MUFA, n-6, and n-3 PUFA. A study based on 60 tree peony cultivars revealed that the main 
fatty acids in tree peony are palmitic acid, stearic acid, oleic acid, linoleic acid, and $\alpha$-linolenic acid, accounting for $4.9-7.1 \%, 0.9-2.1 \%, 14.2-31.6 \%, 19.1-41.1 \%$, and $26.1-54.7 \%$ of fatty acids respectively [2]. Such a high level of $\alpha$-linolenic acid is uncommon in plant seed oils. In line with previous findings, data from our dietary fatty acid profile analysis affirmed high levels of $\alpha$-linolenic acid, linoleic acid, and oleic acid in HFD-PSO, accounting for $40 \%, 27 \%$, and $23 \%$ respectively [2-4]. Intriguingly, our data also suggested that in response to high intake of $\alpha$-linolenic acid in HFD-PSO, n-3 PUFA levels, particularly $\alpha$-linolenic acid, was strongly raised in vivo in the hamster model, while n-6 PUFA was not changed much, resulting in a significant decrease in the ratio of n-6 to n-3 PUFA (Table 3). Differential effects of PSO supplement on in vivo n-3 and n-6 PFUA levels could be due to strong competition on key enzymes for elongation and desaturation reactions between n-3 and n-6 PUFA metabolic pathways. Typical western diets have high ratio of n-6 to n-3 PUFA, ranging between 15:1 and 20:1. Although still inconclusive, high ratios of n-6 to n-3 PUFA are associated with increased risks of different CVD. It's been recommended by different health authorities to reduce dietary ratio of n- 6 to n-3 for heart health benefits [13]. Therefore, increased intake of PSO could be another effective way for this medical purpose.

In our study, PSO also showed differential effects on triglyceride and cholesterol levels, strongly decreasing circulating and adipose triglyceride, while having no effect on both LDL-choleterol and HDL-cholesterol (Figure 2). Interestingly, these findings are somehow different from the recent report by $\mathrm{Su}$ et al [25], which revealed the PSO supplement decreased both triglyceride and total cholesterol (decreased LDL-C and increased HDL-C) in a high fat Wistar rat animal model. Such an apparent discrepancy in cholesterol metabolism by PSO supplement could be due to the different animal model used. As described in introduction, hamsters are much closer in cholesterol metabolism to humans than other rodent species. In contrast to unanimously reported triglyceride-lowering benefit, effects of different unsaturated fatty acids on circulating cholesterol remain inconclusive. Suppression of circulating cholesterol levels by dietary unsaturated fatty acids has been reported in both rodents and human subjects. However, there have been no or even opposite effects on cholesterol level also reported by many animal and clinical studies [26-29]. For example, Terpstra et al demonstrated that increasing unsaturation of dietary fat could lower HDL-cholesterol in hamsters [29]. A systemic review based on 22 clinical studies showed an increase in both LDL and HDL-cholesterol in subjects taking n-3 PUFA over 4 weeks [30]. Echoing with these interesting clinical findings, we showed no effect of PSO supplement on circulating cholesterol levels. Moreover, although decreased HDL cholesterol levels has been long recognized as an important risk factor for CVD, some recent evidence raised questions on heart benefit of HDL-cholesterol, showing association of increased plasma HDL-C and risk of coronary heart disease [31].

In addition to the total fat content in the diet, specific types of fats may play important roles in NAFLD pathophysiology. High intake of saturated fats has been demonstrated to be adverse 
in pathogenesis of NAFLD, while PUFA, especially n-3 PUFA, may be protective against intravenous fat accumulation by enhancing fatty acid oxidation and decreasing lipogenesis. PUFA concentrations have been shown to be lower in patients with NAFLD, and had a less favorable higher ratio of n-6 to n-3 PUFA. Study by Araya et al demonstrated that ratio of n- 6 to n-3 PUFA was correlated with hepatic steatosis in NAFLD patients [16]. A systemic review and meta-analysis based on 9 clinical studies and 355 individuals suggested that while optimal doses are unknown, n-3 PUFA supplement decreased liver fat accumulation and improvement in liver functions [32]. These benefits were also observed in our study, in addition to another study by $\mathrm{Su}$ et al [25]. Therefore, PSO, which is rich in $\alpha$-linolenic acid, could be a good source of n-3 PUFA for NAFLD intervention.

\section{CONCLUSIONS:}

In summary, with a high fat hamster animal model, dietary supplement of PSO rich in different unsaturated fatty acids, especially $\alpha$-linolenic acid, was showed to effectively reduce important risk factors implicated in CVD and NAFLD, providing compelling scientific evidence to support use of this plant edible oil to reduce incidence of some metabolic diseases.

\section{Competing Interests:}

Zhiqiang Zheng, Yingyi Mao and Yan Guan are employees of Abbott Laboratories. All authors have nothing to declare.

\section{Authors' Contributions:}

Z. Z. designed research; Z. Z., Y. M., X. T., and J. H. conducted research; Z. Z., Y. M. and J. H. analyzed data; Z. Z. wrote the paper; Y. G. and Y. H. had primary responsibility for final content. All authors read and approved the final manuscript.

\section{Abbreviations:}

MUFA, monounsaturated fatty acids; PUFA, polyunsaturated fatty acids; HFD, high fat diets; CO, coconut oil; PSO, peony seed oil; NAFLD, non-alcoholic fatty liver disease; CVD, cardiovascular diseases; LDL, low-density lipoprotein; HDL, high-density lipoprotein; H\&E, hematoxylin and eosin; RBC, red blood cells; ANOVA, analysis of variance; SEM, standard error of the mean

\section{Acknowledgments and Funding:}

We are grateful to HD Biosciences (China) Co., Ltd. for providing necessary experimental assistance. This work is financially supported by Abbott Laboratories and National Natural Science Foundation of China (31070617), the Technologies R\&D Program of Shanghai Greenery and Public Sanitation Bureau (G142417, G142420, and G162403). 


\section{REFERENCES}

1. Wang JG, Zhang, Z.S.: Herbaceous Peonies of China. China Forestry Publishing House, Beijing, China; 2005.

2. Li SS, Yuan RY, Chen LG, Wang LS, Hao XH, Wang LJ, Zheng XC, Du H: Systematic qualitative and quantitative assessment of fatty acids in the seeds of 60 tree peony (Paeonia section Moutan DC.) cultivars by GC-MS. Food Chem 2015, 173:133-140.

3. He C, Peng Y, Xiao W, Liu H, Xiao PG: Determination of chemical variability of phenolic and monoterpene glycosides in the seeds of Paeonia species using HPLC and profiling analysis. Food Chem 2013, 138:2108-2114.

4. Li SS, Wang LS, Shu QY, Wu J, Chen LG, Shao S, Yin DD: Fatty acid composition of developing tree peony (Paeonia section Moutan DC.) seeds and transcriptome analysis during seed development. BMC Genomics 2015, 16:208.

5. Qi JCZ, H.M.; Ma, J.Q.; Li, P.: Analysis of the chemical constituents in peony seed oil by GC-MS. Cereals \& Oils 2005, 11:2.

6. Connor WE: Alpha-linolenic acid in health and disease. Am J Clin Nutr 1999, 69:827-828.

7. Siri-Tarino PW, Sun Q, Hu FB, Krauss RM: Saturated fatty acids and risk of coronary heart disease: modulation by replacement nutrients. Curr Atheroscler Rep 2010, 12:384-390.

8. Sullivan S: Implications of diet on nonalcoholic fatty liver disease. Curr Opin Gastroenterol 2010, 26:160-164.

9. Zelber-Sagi S, Nitzan-Kaluski D, Goldsmith R, Webb M, Blendis L, Halpern Z, Oren R: Long term nutritional intake and the risk for non-alcoholic fatty liver disease (NAFLD): a population based study. J Hepatol 2007, 47:711-717.

10. Shahidi F, Miraliakbari H: Omega-3 (n-3) fatty acids in health and disease: Part 1--cardiovascular disease and cancer. J Med Food 2004, 7:387-401.

11. Shahidi F, Miraliakbari H: Omega-3 fatty acids in health and disease: part 2--health effects of omega-3 fatty acids in autoimmune diseases, mental health, and gene expression. J Med Food 2005, 8:133-148.

12. Delpech JC, Madore C, Joffre C, Aubert A, Kang JX, Nadjar A, Laye S: Transgenic increase in n-3/n-6 fatty acid ratio protects against cognitive deficits induced by an immune challenge through decrease of neuroinflammation. Neuropsychopharmacology 2015, 40:525-536.

13. 13. WHO F: Fats and oils in human nutrition: Report of a joint expert consultation. Rome; 1994.

14. 14. Mozaffarian D, Micha R, Wallace S: Effects on coronary heart disease of increasing polyunsaturated fat in place of saturated fat: a systematic review and meta-analysis of 
randomized controlled trials. PLoS Med 2010, 7:e1000252.

15. Di Minno MN, Russolillo A, Lupoli R, Ambrosino P, Di Minno A, Tarantino G: Omega-3 fatty acids for the treatment of non-alcoholic fatty liver disease. World $J$ Gastroenterol 2012, 18:5839-5847.

16. Araya J, Rodrigo R, Videla LA, Thielemann L, Orellana M, Pettinelli P, Poniachik J: Increase in long-chain polyunsaturated fatty acid $n-6 / n-3$ ratio in relation to hepatic steatosis in patients with non-alcoholic fatty liver disease. Clin Sci (Lond) 2004, 106:635-643.

17. Simopoulos AP: The importance of the omega-6/omega-3 fatty acid ratio in cardiovascular disease and other chronic diseases. Exp Biol Med (Maywood) 2008, 233:674-688.

18. Russo GL: Dietary n-6 and n-3 polyunsaturated fatty acids: from biochemistry to clinical implications in cardiovascular prevention. Biochem Pharmacol 2009, 77:937-946.

19. Dillard A, Matthan NR, Lichtenstein AH: Use of hamster as a model to study diet-induced atherosclerosis. Nutr Metab (Lond) 2010, 7:89.

20. Spady DK, Dietschy JM: Dietary saturated triacylglycerols suppress hepatic low density lipoprotein receptor activity in the hamster. Proc Natl Acad Sci U $S$ A 1985, 82:4526-4530.

21. Liu GL, Fan LM, Redinger RN: The association of hepatic apoprotein and lipid metabolism in hamsters and rats. Comp Biochem Physiol A Comp Physiol 1991, 99:223-228.

22. Nistor A, Bulla A, Filip DA, Radu A: The hyperlipidemic hamster as a model of experimental atherosclerosis. Atherosclerosis 1987, 68:159-173.

23. Barcelo-Coblijn G, Murphy EJ, Othman R, Moghadasian MH, Kashour T, Friel JK: Flaxseed oil and fish-oil capsule consumption alters human red blood cell n-3 fatty acid composition: a multiple-dosing trial comparing 2 sources of n-3 fatty acid. Am J Clin Nutr 2008, 88:801-809.

24. Kleiner DE, Brunt EM, Van Natta M, Behling C, Contos MJ, Cummings OW, Ferrell LD, Liu YC, Torbenson MS, Unalp-Arida A, et al: Design and validation of a histological scoring system for nonalcoholic fatty liver disease. Hepatology 2005 , 41:1313-1321.

25. Su J, Ma C, Liu C, Gao C, Nie R, Wang H: Hypolipidemic Activity of Peony Seed Oil Rich in alpha-Linolenic, is Mediated Through Inhibition of Lipogenesis and Upregulation of Fatty Acid beta-Oxidation. J Food Sci 2016, 81:H1001-1009.

26. Egert S, Kannenberg F, Somoza V, Erbersdobler HF, Wahrburg U: Dietary alpha-linolenic acid, EPA, and DHA have differential effects on LDL fatty acid composition but similar effects on serum lipid profiles in normolipidemic humans. $J$ Nutr 2009, 139:861-868. 
27. Oelrich B, Dewell A, Gardner CD: Effect of fish oil supplementation on serum triglycerides, LDL cholesterol and LDL subfractions in hypertriglyceridemic adults. Nutr Metab Cardiovasc Dis 2013, 23:350-357.

28. Goh YK, Jumpsen JA, Ryan EA, Clandinin MT: Effect of omega 3 fatty acid on plasma lipids, cholesterol and lipoprotein fatty acid content in NIDDM patients. Diabetologia 1997, 40:45-52.

29. Terpstra AH, van den Berg P, Jansen H, Beynen AC, van Tol A: Decreasing dietary fat saturation lowers HDL-cholesterol and increases hepatic HDL binding in hamsters. $\mathrm{Br} \mathrm{J}$ Nutr 2000, 83:151-159.

30. Jacobson TA, Glickstein SB, Rowe JD, Soni PN: Effects of eicosapentaenoic acid and docosahexaenoic acid on low-density lipoprotein cholesterol and other lipids: a review. $J$ Clin Lipidol 2012, 6:5-18.

31. Zanoni P, Khetarpal SA, Larach DB, Hancock-Cerutti WF, Millar JS, Cuchel M, DerOhannessian S, Kontush A, Surendran P, Saleheen D, et al: Rare variant in scavenger receptor BI raises HDL cholesterol and increases risk of coronary heart disease. Science 2016, 351:1166-1171.

32. Parker HM, Johnson NA, Burdon CA, Cohn JS, O'Connor HT, George J: Omega-3 supplementation and non-alcoholic fatty liver disease: a systematic review and meta-analysis. J Hepatol 2012, 56:944-951. 